\title{
Geometrical Theory of the Hyperbolic Functions.
}

By W. L. Thomson, M.A.

Figure 1.

1. If PQRS be an Hyperbola, $O E, O F$ its asymptotes, $P, Q, R, S$ any points on it such that the sectorial area $O P Q=$ sectorial area ORS; and if $\mathrm{PA}, \mathrm{QB}, \mathrm{RC}, \mathrm{SD}$ be ordinates to one asymptote and parallel to the other, it is known that

$$
O A: O B=O C: O D \text {, and } P A: Q B=R C: S D
$$

Hence if $A, B, C \ldots$ be taken so that $O A, O B, O C \ldots$ are in continued proportion, the areas $O P Q, O Q R, O R S$... are all equal, and since the number of points can be made as large as we please, the sum of the sectorial areas can be made as large as we please.

$\therefore$ The area between an asymptote, the curve, and any radius vector is infinite.

\section{Figure 2.}

2. Let $P$ be any point on a rectangular hyperbola whose usymptotes ure $O E, O F$ and axis $O A$. Draw $P M$ perpendicular to $\mathrm{OA}$ meeting $\mathrm{OE}$ in $p, \mathrm{~PB}$ perpendicular to $\mathrm{OE}, \mathrm{P} p^{\prime}$ parallel to $\mathrm{OA}$ and $A D$ perpendicular to $O E$.

Then

$$
\widehat{\mathrm{O} p \mathrm{M}}=4 \tilde{a}^{\circ}=\widehat{\mathrm{B}^{\prime} \mathrm{P}} \quad \therefore \mathrm{B} p=\mathrm{BP}=\mathrm{B}^{\prime} .
$$

From similar triangles

$$
\begin{aligned}
\frac{O B+B P}{O M}=\frac{O A}{O D} \quad(2) \quad \text { and } \quad \frac{O B-B P}{P M}=\frac{O A}{O D} \quad(3) ; \\
\therefore \frac{O B+B P}{O A}=\frac{O M}{O D} \quad(4) \quad \text { and } \quad \frac{O B-B P}{O A}=\frac{P M}{O D} \quad(5) .
\end{aligned}
$$

Hence if $Q$ be any other point on the curve, QC, QN perpendicular to asymptote and axis,

$$
\frac{\mathrm{OC}+\mathrm{CQ}}{\mathrm{OA}}=\frac{\mathrm{ON}}{\mathrm{OD}} \quad(6) \quad \text { and } \quad \frac{\mathrm{OC}-\mathrm{CQ}}{\mathrm{OA}}=\frac{\mathrm{QN}}{\mathrm{OD}}
$$

$\therefore$ Multiplying (4) and (6)

$$
\frac{\mathrm{OB} \cdot \mathrm{OC}+\mathrm{BP} \cdot \mathrm{CQ}+\mathrm{OC} \cdot \mathrm{BP}+\mathrm{OB} \cdot \mathrm{CQ}}{\mathrm{OA}}=\frac{\mathrm{OM} \cdot \mathrm{ON}}{\mathrm{OD}^{2}}
$$


but

$$
O A^{2}=2 O D^{2} ;
$$

$\therefore \quad O B \cdot O C+B P \cdot C Q+O C \cdot B P+O B \cdot C Q=2 O M . O N$.

Similarly from (5) and (7)

$O B \cdot O C+B P . C Q-O C \cdot B P-O B \cdot C Q=2 P M . Q N$.

Adding and dividing by 2

$$
\mathrm{OC} \cdot \mathrm{OB}+\mathrm{BP} \cdot \mathrm{CQ}=\mathrm{OM} \cdot \mathrm{ON}+\mathrm{PM}, \mathrm{QN} \text {. }
$$

In the same way by multiplying (4) by (7) and (5) by (6), and adding we get

$$
O B \cdot O C-B P \cdot C Q=O M \cdot Q N+O N \cdot P M
$$

\section{Fradre 3.}

3. Let $P M, O M$ be ordinate and abscissa of any point on the right hand branch of the rectangular hyperbola whose axis is OA. Let $\mathrm{OA}=a$ and area $\mathrm{OAP}=\mathrm{U}$, and let $\frac{2 \mathrm{U}}{a^{2}}=u$.

Then $u$ is our variable and the definitions are

$$
\begin{aligned}
& \sinh u=\frac{\mathrm{PM}}{\mathrm{OA}}, \cosh u=\frac{\mathrm{OM}}{\mathrm{OA}}, \tanh u=\frac{\mathrm{PM}}{\mathrm{OM}}, \\
& \operatorname{coth} u=\frac{\mathrm{OM}}{\mathrm{PM}}, \operatorname{sech} u=\frac{\mathrm{OA}}{\mathrm{OM}}, \operatorname{cosech} u=\frac{\mathrm{OA}}{\mathrm{PM}} .
\end{aligned}
$$

4. The functions so defined are independent of the particular hyperbola we take, that is to say, given $u, \sinh u$, etc., are all determinate.

For all rectangular hyperbolas are similar figures and taking them with the same asymptotes, the centre is the centre of similarity. Then, drawing OPP' cutting any two in $P, P^{\prime}$, (Fig. 3) $P, P^{\prime}$ are corresponding points.

$$
\text { Now if } \quad u=\frac{20 \mathrm{AP}}{\mathrm{OA}^{3}} \text {, it also }=\frac{20 \mathrm{~A}^{\prime} \mathrm{P}^{\prime}}{\mathrm{OA}^{\prime \prime}} \text {, }
$$

since corresponding areas are as the squares of corresponding lines. Also $\mathbf{P M}, \mathbf{P}^{\prime} \mathbf{M}^{\prime}$ are corresponding lines being parallel.

$\therefore \frac{P M}{O A}=\frac{P^{\prime} M^{\prime}}{\mathrm{OA}^{\prime}}$ i.e. $\sinh u$ depends only on $u$.

Sinilarly for the other ratios. 


\section{4}

5. From the definitions we have

$\sinh u=\frac{1}{\operatorname{cosech} u}, \quad \cosh u=\frac{1}{\operatorname{sech} u}, \quad \tanh u=\frac{1}{\operatorname{coth} u}=\frac{\sinh u}{\cosh u}$.

Also from the known property of the rectangular hyperbola that $O M^{2}-P^{3}=\mathrm{OA}^{2}$ we derive the three equations

$\cosh ^{2} u-\sinh ^{2} u=1, \quad \tanh ^{2} u+\operatorname{sech}^{2} u=1, \quad \operatorname{coth}^{2} u-\operatorname{cosech}^{2} u=1$.

6. The signs of lines are determined as in the circular functions, and $u$ is + if OP rotates counterclockwise, - if clockwise.

Hence it is evident that $\sinh u, \tanh u, \operatorname{coth} u$, and $\operatorname{cosech} u$ are odd functions, while $\cosh u$ and $\operatorname{sech} u$ are even functions.

Figure 4.

7. Addition Theorem.

Let $\mathrm{OAP}=\mathrm{U}, \quad \mathrm{OPQ}=\mathrm{U}^{\prime}$.

Make $\mathrm{OAR}=\mathrm{U}^{\prime}$.

Draw $Q M, P L, R N$ perpendicular to the axis,

$\mathrm{QB}, \mathrm{PC}, \mathrm{RE}$ perpendicular to the asymptote.

Then if $u=\frac{2 \mathrm{U}}{a^{2}}$ and $v=\frac{2 \mathrm{U}^{\prime}}{a^{2}}, \quad \frac{\mathrm{QM}}{\mathrm{OA}}=\sinh (u+v), \frac{\mathrm{OM}}{\mathrm{OA}}=\cosh (u+v)$.

Now $\frac{O B-B Q}{O A}=\frac{Q M}{O D}, \therefore \frac{O B-B Q}{2 \overline{O D}}=\frac{Q M}{O A}$, since $O A^{2}=2 O D^{2}$.

$$
\begin{aligned}
& \therefore \quad \frac{\mathrm{OB} \cdot \mathrm{OD}-\mathrm{BQ} \cdot \mathrm{OD}}{2 \mathrm{OD}^{2}}=\frac{\mathrm{QM} \cdot \mathrm{OA}}{\mathrm{OA}^{2}} ; \\
& \begin{aligned}
\therefore \quad \mathrm{QM} \cdot \mathrm{OA} & =\mathrm{OE} \cdot \mathrm{OD}-\mathrm{BQ} \cdot \mathrm{OD} \\
& =\mathrm{OE} \cdot \mathrm{OC}-\mathrm{PC} \cdot \mathrm{RE} \text { by }(1), \\
& =\mathrm{OL} \cdot \mathrm{RN}+\mathrm{ON} \cdot \mathrm{PL} \text { by }(9) . \\
\therefore \quad \frac{\mathrm{QM}}{\mathrm{OA}} & =\frac{\mathrm{OL}}{\mathrm{OA}} \cdot \frac{\mathrm{RN}}{\mathrm{OA}}+\frac{\mathrm{ON}}{\mathrm{OA}} \cdot \frac{\mathrm{PL}}{\mathrm{OA}} ;
\end{aligned}
\end{aligned}
$$

i.e. $\quad \sinh (u+v)=\sinh u \cosh v+\cosh u \sinh v$.

Again

$$
\begin{aligned}
\frac{\mathrm{OE}+\mathrm{BQ}}{2 \mathrm{OD}}=\frac{\mathrm{OM}}{\mathrm{OA}} \text { from } & \\
\therefore \quad & \frac{\mathrm{OB} \cdot \mathrm{OD}+\mathrm{BQ} \cdot \mathrm{OD}}{2 O \mathrm{D}^{2}}=\frac{\mathrm{OM} \cdot \mathrm{OA}}{O \mathrm{OA}^{2}} ;
\end{aligned}
$$




$$
\begin{aligned}
& \therefore \quad \text { OM. OA }=O B \cdot O D+B Q \cdot D O \\
&=O L \cdot O N+R N \cdot P L . \\
& \therefore \quad \frac{O M}{O A}=\frac{O L}{O A} \cdot \frac{O N}{O A}+\frac{R N}{O A} \cdot \frac{P L}{O A} ;
\end{aligned}
$$

i.e. $\cosh (u+v)=\cosh u \cosh v+\sinh u \sinh v$.

Whence also $\sinh (u-v)=\sinh u \cosh v-\cosh u \sinh v$,

and $\cosh (u-v)=\cosh u \cosh v-\sinh u \sinh v$.

Whence also we may obtain formulæ for multiples and sub-multiples of $u$. Whence also we obtain in the usual way

$$
\cosh n u=\cosh ^{n} u+{ }_{n} \mathrm{C}_{2} \cosh ^{n-2} u \sinh ^{2} u+\ldots \text { etc. }
$$

8. Fundamental Inequalities.

\section{Figure 3.}

$$
\begin{aligned}
& \sinh u=\frac{\mathrm{PM}}{\sigma \mathrm{OA}}=\frac{\mathrm{PM} . \mathrm{OA}}{\mathrm{OA}^{2}}=\frac{2 \Delta \mathrm{OAP}}{\mathrm{OA}^{2}}>\frac{2 \text { sectorial area OAP }}{\mathrm{OA}^{2}} \\
& >u \text {. } \\
& \text { Also } \sinh ^{2} u=\cosh ^{2} u-1 \text {. } \\
& \therefore \quad \sinh u<\cosh u \text {. } \\
& \tanh u=\frac{\mathbf{P M}}{\mathrm{OM}}=\frac{\mathbf{A T}}{\mathrm{OA}}, \quad \text { (if } \mathbf{A T} \text { is the tangent at } \mathbf{A} \text { ), } \\
& =\frac{\mathrm{AT} \cdot \mathrm{OA}}{\mathrm{OA}^{2}}=\frac{2 \Delta \mathrm{OAT}}{\mathrm{OA}^{2}} \\
& <\frac{2 \text { sectorial area OAP }}{\mathrm{OA}^{2}} \\
& <u \text {. } \\
& \therefore \quad \tanh u<u<\sinh u<\cosh u-\quad-\quad- \\
& \text { Also } \sinh u=2 \sinh \frac{u}{2} \cosh \frac{u}{I} \\
& =2 \tanh \frac{u}{2} / \operatorname{sech}^{2} \frac{u}{2} \\
& =\frac{2 \tanh \frac{u}{2}}{1-\tanh ^{2} \frac{u}{2}} \\
& <\frac{2 \cdot \frac{u}{2}}{1-\left(\frac{u}{2}\right)^{2}}<\frac{u}{1-\frac{u^{2}}{4}} \text {, since } u>\tanh u \text {. } \\
& \cosh \frac{u}{2}=\frac{1+\tanh ^{2} \frac{u}{2}}{1-\tanh ^{2} \frac{u}{2}}<\frac{1+\frac{u^{2}}{4}}{1-\frac{u^{2}}{4}}
\end{aligned}
$$


9. Limits.

From (12) $\frac{1}{\cosh u}<\frac{u}{\sinh u}<1, \quad \therefore \underset{u=0}{\text { Lt. }} \frac{u}{\sinh u}=1$.

$\therefore \quad$ Also $\operatorname{Lt}_{u=0} \frac{u}{\tanh u}=1$.

Again

$$
\begin{aligned}
\frac{u}{n} & <\sinh \frac{u}{n}<\frac{\frac{u}{n}}{1-\frac{u^{2}}{4 n^{2}}}, \quad \text { by }(12) \text { and }(13), \\
\therefore \quad 1 & <\frac{\sinh \frac{u}{n}}{\frac{u}{n}}<\frac{1}{1-\frac{u_{2}}{4 n 2}} . \\
\therefore \quad 1 & <\left(\frac{\sinh \frac{u}{n}}{\frac{n}{n}}\right)^{n}<\left(1-\frac{u^{2}}{4 n^{2}}\right)^{-n} .
\end{aligned}
$$

Now $\begin{aligned} \operatorname{Lt}_{n=\infty}\left(1-\frac{u^{2}}{4 n^{3}}\right)^{-n} & =\operatorname{Lt}_{n=\infty}\left\{\left(1-\frac{u^{2}}{4 n^{2}}\right)^{-\frac{4 n^{2}}{u^{2}}}\right\}^{\frac{n^{2}}{4 n}} \\ & =e^{0}=1 .\end{aligned}$

$$
\therefore \quad \operatorname{Lt.}_{n=\infty}\left(\frac{\sinh _{n}^{n}}{\frac{n}{n}}\right)^{n}=1 \quad-\quad-\quad-
$$

$$
\begin{array}{rl}
\text { Again } 1 & 1<\cosh \frac{u}{n}<\frac{1+\frac{u^{2}}{4 n^{2}}}{1-\frac{u^{2}}{4 n^{2}}} \\
\therefore \quad 1 & <\left(\cosh \frac{u}{n}\right)^{n}<\left(1+\frac{u_{2}}{4 n^{2}}\right)^{n}\left(1-\frac{u^{2}}{4 n^{2}}\right)^{-n} .
\end{array}
$$

The Limit of the last expression

$$
\begin{aligned}
& =\operatorname{Lt}_{n=\infty}\left\{\left(1+\frac{u^{2}}{4 n^{2}}\right)^{\frac{4 n^{2}}{u^{2}}}\right\}^{\frac{u^{2}}{4 n}} \times \operatorname{Lt}_{n=\infty}\left\{\left(1-\frac{u^{2}}{4 n^{2}}\right)^{-\frac{4 n^{2}}{n^{2}}}\right\}^{\frac{u^{2}}{4 n}} \\
& =e^{0} \times e^{0}=1 . \\
& \therefore \operatorname{Lt}_{n=\infty}\left(\cosh \frac{u}{n}\right)^{n}=1
\end{aligned}
$$


10. From these inequalities we can get the expansion of cosh $u$ and $\sinh u$ in terms of $u$, by the same method as is used in the case of the circular functions, e.g. for $\cosh u$.

From (10) we have

$\cosh n u=\cosh ^{n} u+\frac{n(n-1)}{1.2} \cosh ^{n-2} u \sinh ^{2} u+\ldots$

$$
=\cosh ^{n} u\left\{1+\frac{n(n-1)}{1.2} \tanh ^{2} u+\frac{n(n-1)(n-2)(n-3)}{1.2 .3 .4} \tanh ^{4} u+. .\right\} \text {. }
$$

From the way we got this $n$ must be an integer and the series terminates, but we may take $n$ as large as we please.

Writing $u$ for $n u$ we have

$\cosh u=\cosh ^{n} \frac{u}{n}\left\{1+\frac{n(n-1)}{1.2} \tanh ^{2} \frac{u}{n}+\frac{n(n-1)(n-2)(n-3)}{1.2 .3 .4} \tanh ^{4} \frac{u}{n}+\ldots\right\}$

$=\cosh ^{n} \frac{n}{n}\left\{1+\frac{1\left(1-\frac{1}{n}\right)}{1.2} u^{2} \frac{\tanh ^{2} \frac{u t}{n}}{\left(\frac{n}{n}\right)^{2}}+\frac{1\left(1-\frac{1}{n}\right)\left(1-\frac{2}{n}\right)\left(1-\frac{3}{n}\right)}{1.2 .3 .4} u^{4} \frac{\tanh ^{4} \frac{n}{w}}{\left(\frac{u}{n}\right)^{4}} \cdots\right\}$

$=\cosh ^{n} \frac{n}{n}\left\{1+v_{2}+v_{1} \ldots+v_{2 r}+\mathbf{R}\right\}, r$ being a fixed finite number.

Here $\mathrm{R}=\frac{1\left(1-\frac{1}{n}\right)\left(1-\frac{2}{n}\right) \cdot\left(1-\frac{2 r-1}{n}\right)}{2 r} \cdot u^{2 r}\left(\frac{\tanh \frac{u}{n}}{\frac{u}{u}}\right)^{2 r}+\ldots \quad \begin{gathered}\text { a terminating } \\ \text { series, }\end{gathered}$

$<\frac{1\left(1-\frac{1}{n}\right)\left(1-\frac{2}{n}\right) \ldots\left(1-\frac{2 r-1}{n}\right)}{1.2 .} u^{2 r}+\ldots \quad$ a terminating series,

$<\frac{1}{\frac{\mid 2 r}{2}} u^{2 r}+\frac{1}{\frac{\mid 2 r+2}{\frac{\mid 2 r}{2}}} u^{2 r+2}+\ldots$ to a finite number of terms,

$<\frac{1}{\underline{\mid 2 r}} u^{2 r}+\frac{1}{\underline{\underline{\mid 2 r+2}}} u^{2 r+2}+\ldots$ ad infinitum

$<\frac{u^{2 r}}{\mid 2 r}\left\{1+\frac{u^{2}}{(2 r+1)(2 r+2)}+\frac{u^{4}}{(2 r+1)(2 r+2)(2 r+3)(2 r+4)}+\ldots \underset{i n f}{a d}\right\}$

$<\frac{u^{2 r}}{\underline{\mid 2 r}}\left\{1+\frac{u^{2}}{(2 r+1)^{2}}+\frac{u^{4}}{(2 r+1)^{4}}+\ldots \quad\right.$ ad infinitum $\}$

$<\frac{u^{2 r}}{\underline{\mid 2 r}} \cdot \frac{1}{1-\frac{u^{2}}{(2 r+1)^{2}}}$.

This is true for all values of $n, n$, of course, being $>2 r$. 
Hence it is true when $n$ is infinite.

But

$\underset{n=\infty}{\text { Lt. }}\left(\cosh \frac{n}{n}\right)^{n}=1$

and $\underset{n=\infty}{L}$ L. $\quad v_{2 m}=\frac{u^{2 m}}{\underline{\mid 2 m}}, \quad m$ being finite.

$\therefore$ Since $r$ is finite

$$
\cosh u=1+\frac{u^{2}}{2 !}+\frac{u^{4}}{4 !}+\quad+\frac{u^{2 r}}{2 r !}+\mathrm{R}_{2 r},
$$

where $R_{2 r}$ is subject to condition (17).

Now $\quad \operatorname{Lt}_{r=\infty} \frac{u^{2 r}}{\underline{\mid 2 r}} \cdot \frac{1}{1-\frac{u^{2}}{(2 r+1)^{2}}}=0$

$$
\therefore \quad \text { Lt. } \mathbf{R}_{2 r}=0 \text {. }
$$

Hence we may write $\cosh u=1+\frac{u^{2}}{2 !}+\frac{u^{4}}{4 !}+\ldots$ ad infinitum.

In the same way we get

$$
\begin{aligned}
& \text { whence } \begin{aligned}
\sinh u & =u+\frac{u^{3}}{3 !}+\frac{u^{5}}{5 !}+\ldots \text { ad infinitum. } \\
\cosh u & =\frac{e^{u}+e^{-u}}{2}, \\
\text { and } \quad \sinh u & =\frac{e^{u}-e^{-u}}{2}, \quad \text { the usual definitions. }
\end{aligned}
\end{aligned}
$$

